\title{
Optical properties of single-layer and bilayer arsenene phases
}

\author{
D. Kecik, ${ }^{1}$ E. Durgun, ${ }^{1,2,{ }^{*}}$ and S. Ciraci ${ }^{3, \dagger}$ \\ ${ }^{1}$ UNAM-National Nanotechnology Research Center, Bilkent University, Ankara 06800, Turkey \\ ${ }^{2}$ Institute of Materials Science and Nanotechnology, Bilkent University, Ankara 06800, Turkey \\ ${ }^{3}$ Department of Physics, Bilkent University, Ankara 06800, Turkey
}

(Received 8 September 2016; published 8 November 2016)

\begin{abstract}
An extensive investigation of the optical properties of single-layer buckled and washboard arsenene and their bilayers was performed, starting from layered three-dimensional crystalline phase of arsenic using density functional and many-body perturbation theories combined with random phase approximation. Electron-hole interactions were taken into account by solving the Bethe-Salpeter equation, suggesting first bound exciton energies on the order of $0.7 \mathrm{eV}$. Thus, many-body effects were found to be crucial for altering the optical properties of arsenene. The light absorption of single-layer and bilayer arsenene structures in general falls within the visible-ultraviolet spectral regime. Moreover, directional anisotropy, varying the number of layers, and applying homogeneous or uniaxial in-plane tensile strain were found to modify the optical properties of two-dimensional arsenene phases, which could be useful for diverse photovoltaic and optoelectronic applications.
\end{abstract}

DOI: 10.1103/PhysRevB.94.205410

\section{INTRODUCTION}

The intriguing structural, electronic, and optical properties of many two-dimensional (2D) materials similar to graphene such as the transition-metal dichalcogenides [1-3], group IV elements [4,5], and group III-V based 2D materials [6-8] have motivated further research for new discoveries. Owing to its attractive intrinsic properties such as high carrier mobility, direct band gap, and superior optical properties, phosphorene has been widely in focus [9-12]. The layered black and blue phosphorus with band gaps suitable for $2 \mathrm{D}$ electronics have initiated the search for similar structures in other group $\mathrm{V}$ elements or pnictogens. In general, the recent theoretical works have predicted that nitrogen [13], phosphorus [14], arsenic [15], antimony [16,17], and bismuth [18] can form similar single-layer, buckled honeycomb or washboard structures.

Diverse allotropes of arsenene have been studied more with a focus on their structural, mechanical, and electronic properties, where arsenene and antimonene were also found to display high carrier mobility, superior mechanical properties, negative Poisson's ratio, and possible topological phase transition features in themselves [19-27]. The layered three-dimensional (3D) phase of arsenic, i.e., gray arsenic, presents strong evidence that single-layer arsenic can exist as a local minima in the Born-Oppenheimer surface. In fact, Kamal and Ezawa [15], based on density functional theory (DFT) calculations [28], achieved optimizing single layers of buckled honeycomb (b-As), as well as of washboard ( $w$-As) structures of arsenic, named as arsenene. They also showed that these structures are stable against the long-wavelength displacements indicating the stability at $T=0 \mathrm{~K}$. The semiconducting character having a band gap in the range of $1.5-2.10 \mathrm{eV}$ and other properties of arsenene have attracted considerable interest in flexible 2D nanoelectronics, optoelectronics, photocatalytic applications, and nanomaterial fabrication. Now, efforts have focused to synthesize these phases [29].

\footnotetext{
*durgun@unam.bilkent.edu.tr

†ciraci@fen.bilkent.edu.tr
}

This paper presents a comprehensive analysis of the optical properties of single-layer (SL) $b$-As and $w$-As and their bilayers (BL) in equilibrium and under uniaxial and biaxial tensile strain. The analysis is extended to $3 \mathrm{D}$ gray arsenic to reveal the dimensionality effects. Based on random phase approximation (RPA), we calculated the imaginary part of the frequency-dependent dielectric function $\left[\varepsilon_{2}(\omega)\right]$ as a function of the photon energy $\hbar \omega$ for lateral $x, y$, and specular $z$ directions. Also, the variation of $\varepsilon_{2}(\omega)$ with applied biaxial and uniaxial strain $\epsilon$ was considered. These calculations were carried out in terms of the electronic structure calculated by standard DFT methods and corrected by state-of-theart techniques such as hybrid functionals and many-body perturbation theory. In addition, we calculated the absorbance, imaginary optical conductivity, optical absorption coefficient, and electron energy loss spectrum for SL, BL, and 3D gray arsenic for incident light polarization along different directions. Finally, the Bethe-Salpeter equation (BSE) is solved to include the electron-hole interactions, hence, the excitonic effects are taken into account by comprising the many-body effects.

The main outcomes of this study can be summarized as follows: (i) The optical response of single-layer arsenene phases is found to be particularly prominent over the solar regime, comprising a window within the visible and ultraviolet (UV) light range. (ii) $2 \mathrm{D}$ form of arsenic is an optically anisotropic material, similar to the aforementioned phosphorene. (iii) Advancing first-principles methods, going from PBE-RPA to $G_{0} W_{0}$-RPA, the optical absorption spectra of SL arsenene were blue-shifted. (iv) The optical properties of layered arsenene are both thickness and tensile strain sensitive. (v) Significant exciton binding energy of SL arsenene phases is revealed.

\section{METHOD}

Electronic structure calculations, which are the preliminary step in our study, were performed in order to investigate the ground-state properties of 3D arsenic and 2D arsenene phases, namely $b$-As and $w$-As and their bilayer structures, within spin-polarized DFT. The projector-augmented-wave 
potentials (PAW) formalism [30,31] implemented in the Vienna $a b$ initio simulation package (VASP) [32-35] was used. The electron exchange and correlation potential was described by the Perdew-Burke-Ernzerhof (PBE) form within the generalized gradient approximation (GGA) $[36,37]$. The plane-wave basis set was used with an energy cutoff of $500 \mathrm{eV}$ for all calculations. Moreover, the van der Waals interactions were accounted for the layered structures $[38,39]$. Atomic positions were optimized using the conjugate gradient method by minimizing the total energy and atomic forces. The energy difference between the sequential steps was set to $10^{-5} \mathrm{eV}$ for convergence. The maximum allowed force on each atom and Gaussian smearing factor were taken as $0.05 \mathrm{eV} / \AA$ and $0.05 \mathrm{eV}$, respectively. $\Gamma$-centered $47 \times 47 \times 1$ and $35 \times 35 \times$ 1 grids were used for Brillouin zone (BZ) integrations for the primitive unit cell of $b$-As and $w$-As, respectively. In order to avoid spurious interactions between the periodic images, a supercell with minimum $\sim 18 \AA$ vacuum space was adopted.

Subsequent to the DFT results, hybrid functionals (HSE06) [40-42] and the quasiparticle (QP) $G_{0} W_{0}$ approach [(QP) $\left.G_{0} W_{0}\right]$ [43-45], where $G$ and $W$ are iterated once, were used to obtain the corrected band structures of $b$-As and $w$-As.

In order to compute the optical properties, RPA [46,47] was employed on top of the PBE approach, using a total of 96 and 192 (valence and conduction) bands, for PBE-RPA and (QP) $G_{0} W_{0}$-RPA calculations, respectively, of which the convergence has been tested accordingly. A Monkhorst-Pack [48] k-point sampling of $127 \times 127 \times 1$ was used for PBE-RPA calculations of $2 \mathrm{D}$ phases. Due to the computational workload, k mesh was reduced to $19 \times 19 \times 1$ and $13 \times 13 \times 1$ for the $b$ - and $w$-As, respectively, while calculating the quasiparticle band energies. Moreover, the k-point sampling and number of bands were scaled accordingly for both 3D arsenic and 2D arsenene structures. The screening was evaluated at 240 frequency points for a majority of the (QP) $G_{0} W_{0}$ calculations. The plane-wave cutoff was decreased to $350 \mathrm{eV}$ and basis set for the response functions was set to $240 \mathrm{eV}$ for the many-body calculations. Local field effects were accounted for both at the levels of Hartree and exchange-correlation potential.

Regarding the optical response beyond the independentparticle approach, the electron-hole interactions were taken into account by solving the BSE [49-51] within the TammDancoff approximation, with the same k-point sampling, energy cutoff, and number of bands setting as in the $(\mathrm{QP}) G_{0} W_{0}$ calculation [52]. The excitonic effects were considered by undertaking the six highest occupied valence and six lowest unoccupied conduction bands.

While the main optical response function is the $\varepsilon_{2}(\omega)$ for light polarized along different spatial directions, several other linear optical spectral quantities can be obtained. Few examples to these spectral functions are the layer-dependent absorbance $[A(\omega)]$, optical conductivity $[\sigma(\omega)]$, absorption coefficient $[\alpha(\omega)]$, and electron energy loss function $\left[-\operatorname{Im}\left(\varepsilon^{-1}\right)\right]$ (see Supplemental Material [53] for the corresponding descriptions of these spectral functions).

\section{SINGLE-LAYER AND BILAYER PHASES OF ARSENENE}

Present study on the optical properties of arsenene phases is based on the structures optimized by PBE calculations

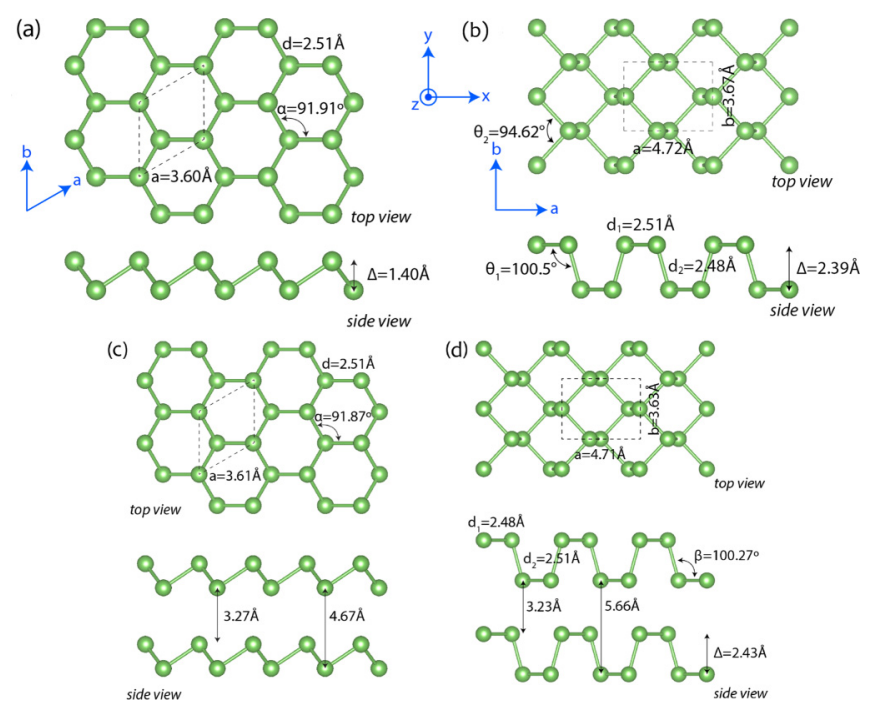

FIG. 1. The atomic structures of SL arsenene phases and their bilayers: (a) Top and side views of SL buckled honeycomb structure $b$-As with its 2D hexagonal lattice. Lattice constants $a$ and $b$, buckling $\Delta$ are given. (b) Same for SL washboard structure $w$-As with 2D rectangular lattice. (c) Top and side views of bilayer of $b$-As and (d) bilayer $w$-As with relevant structural parameters are shown.

(the stability of which were revealed from $a b$ initio phonon and finite-temperature molecular dynamics calculations) and corresponding electronic energy band structures [54]. In addition, elastic properties of SL phases, as well as the response of the electronic structure to uniaxial and biaxial uniform tensile strain, were also treated [54]. In Fig. 1, the atomic structures together with relevant structural parameters of SL and BL $b$-As and $w$-As are presented.

In bilayers of $b$-As and $w$-As, the stacking geometry of the single layers is crucial. For both phases, the minimum of the total energies attained for $A A$ stacking is shown in Figs. 1(c) and 1(d). The $A B$ stacking was found to be energetically less favorable for both structures [54]. Regarding their electronic properties, the direct and indirect band gaps of SL and BL $b$-As and $w$-As phases were computed and presented in Ref. [54]. Accordingly, the band gaps of $b$-As and $w$-As were reported as indirect (direct): 1.59 (1.94) and $0.80(0.90) \mathrm{eV}$, respectively. While bilayer $b$-As has an indirect (direct) gap of $0.70(1.40) \mathrm{eV}, \mathrm{BL} w$-As was found to be semimetallic. In the following sections, the results regarding the optical properties of arsenene will be presented.

\section{OPTICAL PROPERTIES OF ARSENENE PHASES}

Efficient light absorber materials are required for various photovoltaic and optoelectronic applications to operate over a wide energy window, which demand appropriate optical gap of that material. Since monolayer phases of arsenene were found to be promising due to their indirect and direct band gaps which are resonant with the vicinity of the solar regime, their optical properties were vastly investigated in this study.

Similar to the electronic structure calculations presented beforehand, we undertook the approach of employing gradually advancing first-principles methodologies in order to compute the linear optical response of SL and BL arsenene phases. 
All calculations were performed without the inclusion of spin-orbit coupling (SOC) effects since the change in the optical properties of arsenene were observed to be negligible (see Supplemental Material for details [53]). The (QP) $G_{0} W_{0^{-}}$ RPA method was also applied on SL arsenene, where in the final section BSE on top of the $(\mathrm{QP}) G_{0} W_{0}$ approach was undertaken in order to account for the electron-hole interactions. Aside from aiming at acquiring accurately the optical response of SL arsenene structures, we also report how the optical properties are altered once the layer number is increased, in comparison to the properties of layered 3D As.

The optical absorption spectra are presented for the buckled and washboard SL and BL arsenene structures, either with incident light polarized perpendicular to the $c$ axis (i.e., polarized along $x$ or $y$ directions) or parallel to the $c$ axis (i.e., polarized along the $z$ direction), for advancing levels of computational methods, starting from PBE, followed by HSE and (QP) $G_{0} W_{0}$ calculations. See Fig. 1 for visualization of the $x, y, z$ directions and $\mathbf{a}, \mathbf{b}, \mathbf{c}$ lattice vectors.

\section{A. Optical properties of single-layer arsenene}

The $\varepsilon_{2}(\omega)$ of SL $b$-As is displayed in Fig. 2, with PBE, HSE, and $G_{0} W_{0}$-RPA spectra given altogether on the same graph. Accordingly, in general the optical absorption takes place within the spectral range of $2-8 \mathrm{eV}$ for the light polarized along $x$ and $y$ directions and from 4 to $9 \mathrm{eV}$ for the $z$ direction. As for

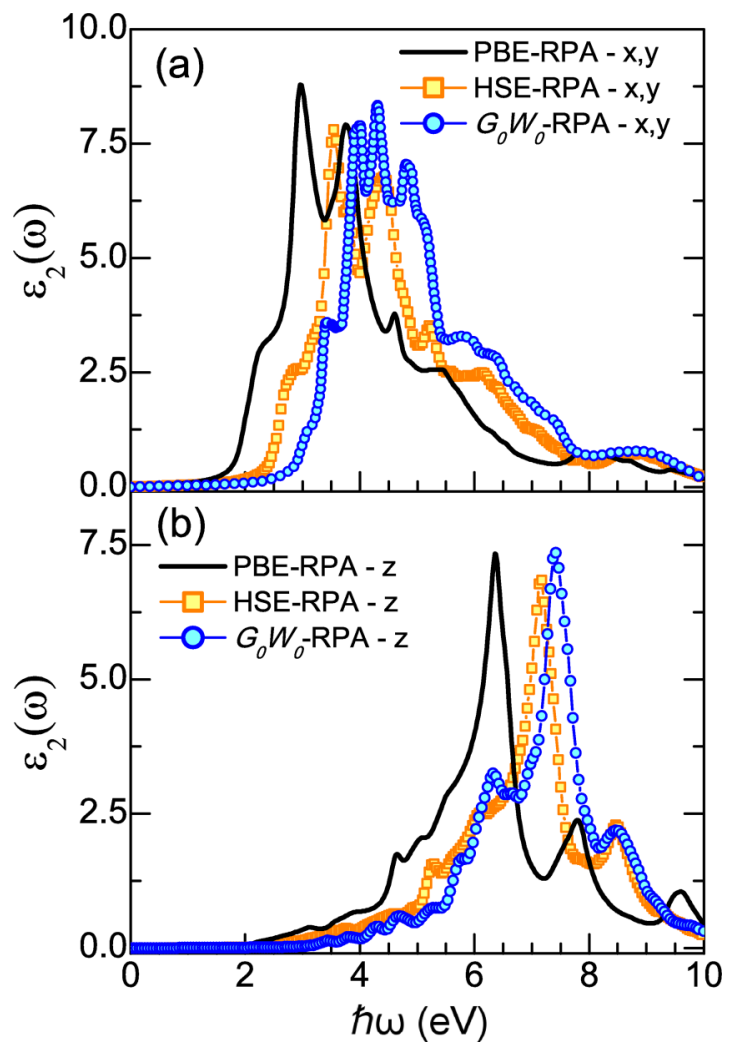

FIG. 2. The optical absorption spectra of SL $b$-As: imaginary dielectric function $\left[\varepsilon_{2}(\omega)\right]$ of SL $b$-As with light polarized (a) along the $x$ and $y$ directions and (b) along the $z$ direction. $G_{0} W_{0}$-RPA spectra are denoted by blue dotted lines, HSE-RPA by orange squared lines, and PBE-RPA results are shown by bold black lines. the PBE-RPA spectrum, the interband optical absorption has an onset prior to $2 \mathrm{eV}$, in compliance with the (non-SOC) band gap of $\sim 1.60 \mathrm{eV}$, where the direct gap was found as $1.94 \mathrm{eV}$. The HSE-RPA approach blue-shifts the PBE-RPA spectrum uniformly by nearly $0.5 \mathrm{eV}$, owing to its band gap of $2.25 \mathrm{eV}$. Furthermore, $G_{0} W_{0}$-RPA spectrum onset is blue-shifted to $\sim 2.6 \mathrm{eV}$. The $G_{0} W_{0}$-RPA imaginary dielectric function points to the main absorption peak focused in the range of $4-6 \mathrm{eV}$. The prominent optical absorption features within this range are mainly dominated by the interband transitions beyond $4 \mathrm{eV}$ at the $M$ high-symmetry point [54]. Hence, in regard to $b$-As, the optical absorption phenomenon is noteworthy within the visible to UV light range. Regarding the case where light is polarized along $z$, the main peak is observed near 6.2 and $7.6 \mathrm{eV}$ for the PBE- and $G_{0} W_{0}$-RPA calculations, respectively. In general, the line shape of the spectra is preserved among the different methodologies.

The anisotropy along the directions of $\mathbf{a}$ and $\mathbf{b}$ lattice vectors within the washboard lattice is significant also regarding its optical properties, as shown in Fig. 3. Absorption onset appears earlier for $w$-As than $b$-As, for light polarized along the $x$ direction. The $G_{0} W_{0}$-RPA spectrum possesses an onset beyond $1 \mathrm{eV}$. The highly anisotropic nature of the washboard structure resembles another member of group $\mathrm{V}$, phosphorene, which is renown for its anisotropic properties, direct band gap, and pseudo-one-dimensional (pseudo-1D) excitonic features [11,55]. While polarization along the $y$ direction for $w$-As and $b$-As allows for the light absorption as of the end of visible spectrum towards the UV range, it is absorbent along the $x$ direction earlier in the spectrum, across all of the visible light range. The $G_{0} W_{0}$-RPA spectrum onset falls prior to $3 \mathrm{eV}$, absorption extending toward $7 \mathrm{eV}$. In general, the light absorption is pronounced in the width range of 4-6 eV for $x$ and $y$ polarizations [see Figs. 3(a) and $3(\mathrm{~b})$ ]. Moreover, the direct band gaps computed using PBE and HSE, found as 0.90 and $1.58 \mathrm{eV}$, are especially in compliance with their respective absorption onsets in $\varepsilon_{2}(\omega)$. Aside from the smallest direct transitions in its band structure, significant interband transitions beyond $3.3 \mathrm{eV}$ are noteworthy around $S$ high-symmetry point [54] within $w$-As, leading to the strong optical peaks. In addition, for light polarized along $z$, the absolute position of the main peak of the HSE and $G_{0} W_{0}$-RPA spectra is concentrated near $6.7 \mathrm{eV}$ [see Fig. 3(c)].

It is worth mentioning that especially the $\varepsilon_{x x}(\omega)$ component of the imaginary dielectric function of $w$-As points to a wide energy window of absorption, which can be a good basis for the design of optical modulator and photovoltaic and optoelectronic materials/devices. Moreover, for both of the SL arsenene structures, when light is polarized along the $x$ or $y$ direction, the common attribute is that light is substantially absorbed between nearly 4 and $7 \mathrm{eV}$, displaying concentrated peaks within the limits of this spectral range.

\section{B. Strain-dependent optical properties}

The optical properties of SL As phases were also investigated under the applied in-plane tensile strain. Biaxial strain applied to SL $b$-As expands the lattice uniformly as introduced in Ref. [54]. The optical response to the uniform lattice expansion is expressed homogeneously in terms of 


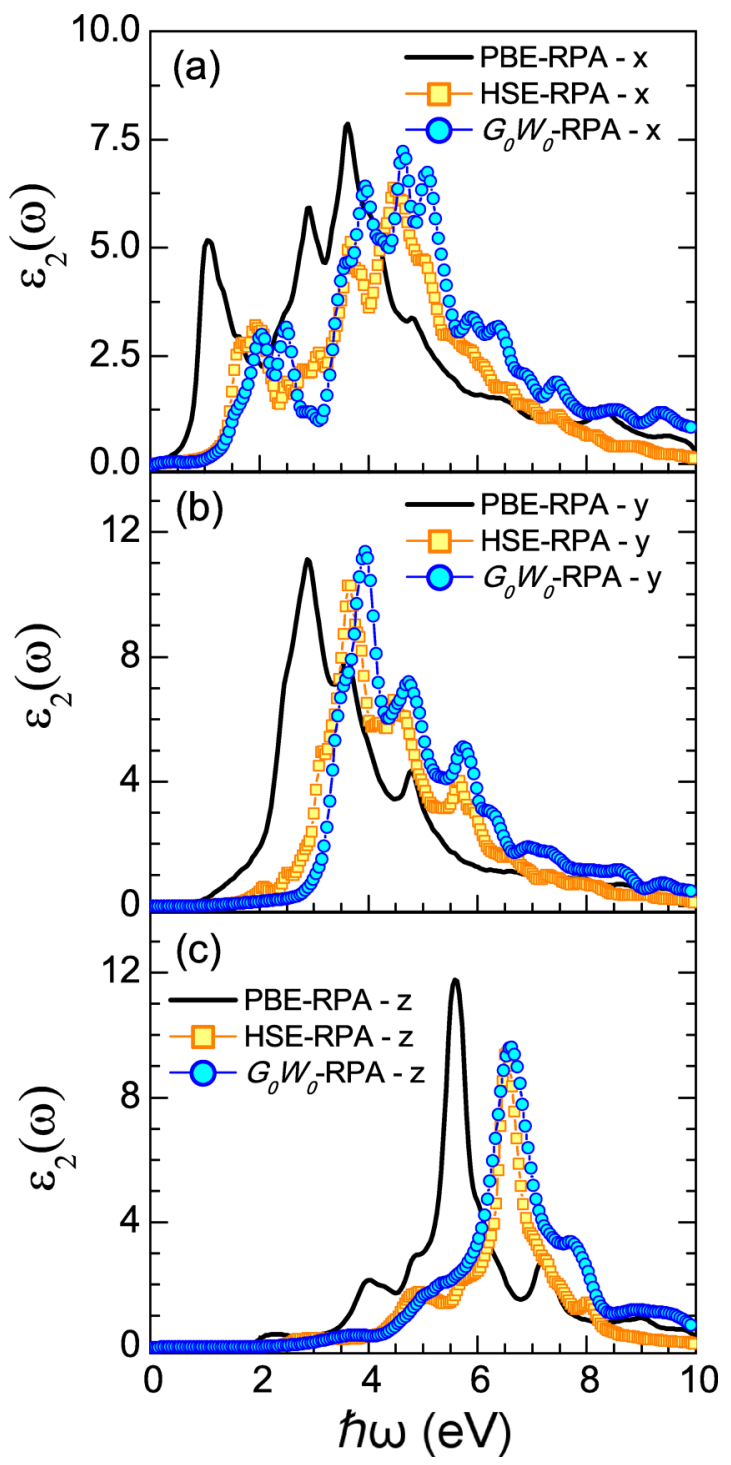

FIG. 3. The optical absorption spectra of SL $w$-As: imaginary dielectric function $\left[\varepsilon_{2}(\omega)\right]$ of SL $w$-As with light polarized (a) along the $x$ direction $\|$ a lattice vector, (b) along the $y$ direction $\| \mathbf{b}$ lattice vector, and (c) along the $z$ direction. $G_{0} W_{0}$-RPA spectra are denoted by blue dotted lines, HSE-RPA by orange squared lines, and PBERPA results are shown by bold black lines.

the isotropic $\varepsilon_{2}(\omega)$, i.e., $x$ and $y$ directions in the dielectric tensor are identical. On the other hand, for $w$-As, uniaxial tensile strain is applied along either lattice vector $\mathbf{a}$ or $\mathbf{b}$. We hereby present the optical response to the former case in terms of the " $x x$ " component of the imaginary dielectric function $\left\{\operatorname{Im}\left[\varepsilon_{x x}(\omega)\right]\right\}$ and the latter in terms of the " $y y$ " component of the imaginary dielectric function $\left\{\operatorname{Im}\left[\varepsilon_{y y}(\omega)\right]\right\}$.

The discussion on the strain and layer-dependent (in the next section) optical properties is based only on the PBE-RPA approach which is seen sufficient for qualitative comparisons. As can be observed from Fig. 4, uniaxial or biaxial tensile strain red-shifts the imaginary dielectric function at uniform incremental intervals. This phenomenon is observed both in $b$-As and $w$-As (when strain applied along lattice vector $\mathbf{b}$ for the latter). For $w$-As, the rigid shifts in $\operatorname{Im}\left[\varepsilon_{x x}(\omega)\right]$ and

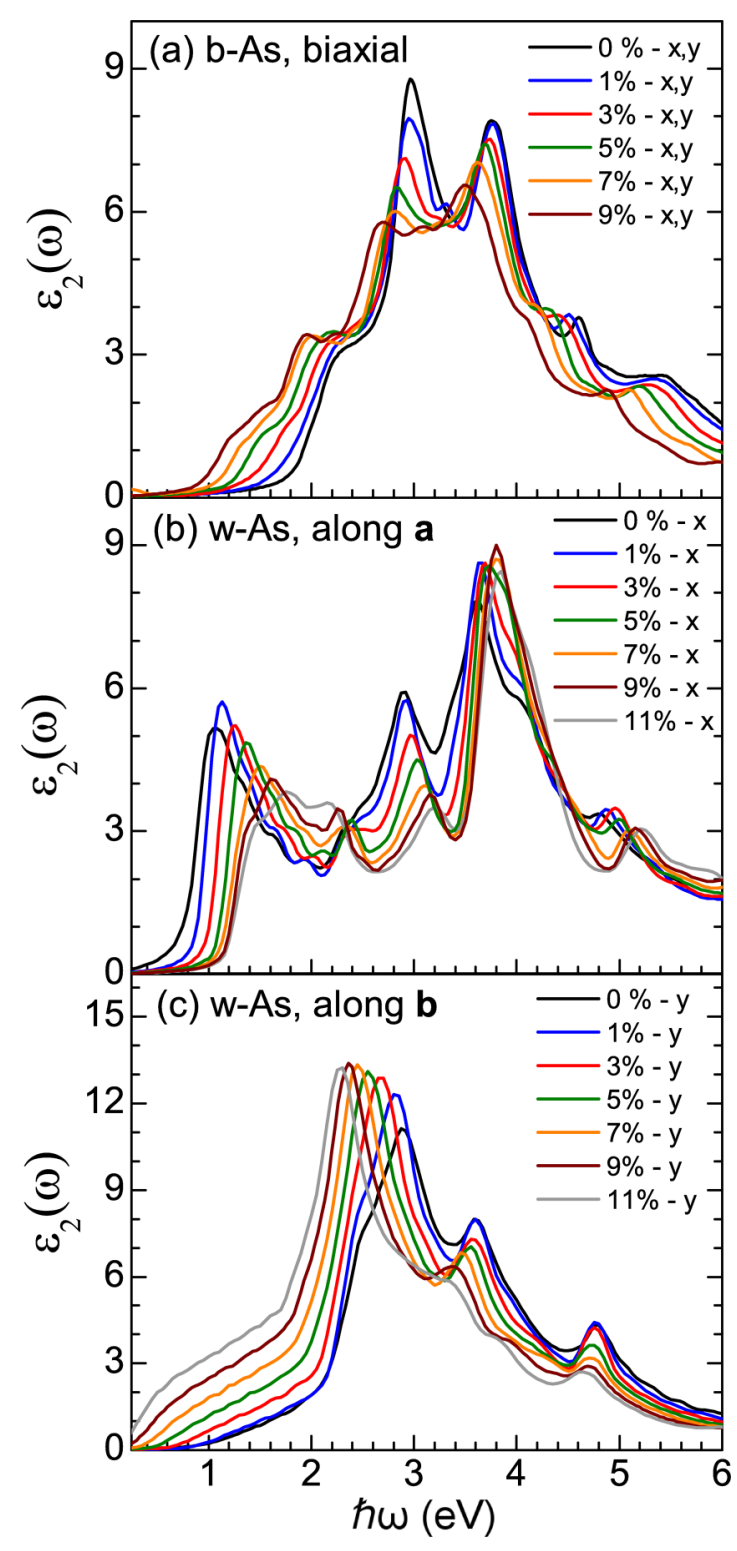

FIG. 4. The optical absorption spectra of SL $b$-As and $w$-As under tensile strain. (a) Imaginary dielectric function $\left[\varepsilon_{2}(\omega)\right]$ of SL $b$-As under biaxial strain. (b) $\varepsilon_{x x}$ component of the imaginary dielectric function of SL $w$-As with strain applied along lattice vector a, and (c) $\varepsilon_{y y}$ component of the imaginary dielectric function of SL $w$-As with strain applied along lattice vector $\mathbf{b}$.

$\operatorname{Im}\left[\varepsilon_{y y}(\omega)\right]$ are more evident when strain is applied along the $a$ and $b$ axes, respectively. In contrast, when strain is applied along the $a$ axis, the optical response along the $x$ direction is opposite to what is generally observed otherwise. The bandgap closing pattern is repeated also for $\varepsilon_{2}(\omega)$ as the onsets are red-shifted to 0 at high levels of strain. These phenomena are related with the variation of the band gap [54], as the direct band gaps of buckled and washboard (for uniaxial strain along b) arsenene phases globally decrease under increasing levels of in-plane tensile strain, in contrast to the $a$-axis strain component of $w$-As. While the aforementioned red-shift in the optical absorption onset of the buckled phase is nearly $1 \mathrm{eV}$ upon $9 \%$ tensile strain, the onset blue-shift of the washboard 
phase introduced by $11 \%$ strain applied along $\mathbf{a}$ is around $0.6 \mathrm{eV}$

\section{Layer-dependent optical properties}

The intensity of $\varepsilon_{2}(\omega)$ for the bilayer phases has increased, for light polarized along the $x$ and $y$ directions; see Figs. 5 and 6 for the absorption spectra of buckled and washboard structures, respectively. It is worth noting that the increase in absorption intensity of BL $w$-As is more pronounced with respect to the buckled phase. The comparison to bulk (gray) arsenic is shown by a ghost plot in gray color, which is scaled accordingly. The onsets of absorption have not been altered significantly arising from the negligible difference of the SL and BL band gaps, although this is not valid for the $z$ direction, pointing to a prominent red-shift with respect to the single-layer spectra, hence, an earlier absorption within the visible regime. The lower-frequency absorption of bulk As can be attributed to its metallic properties, especially to the presence of intraband transitions and collective excitations. While there is significant optical absorption in the visible range, it seems to damp toward $4 \mathrm{eV}$. In summary, when considering the layered structures of arsenic from the aspect of optical applications, the respective

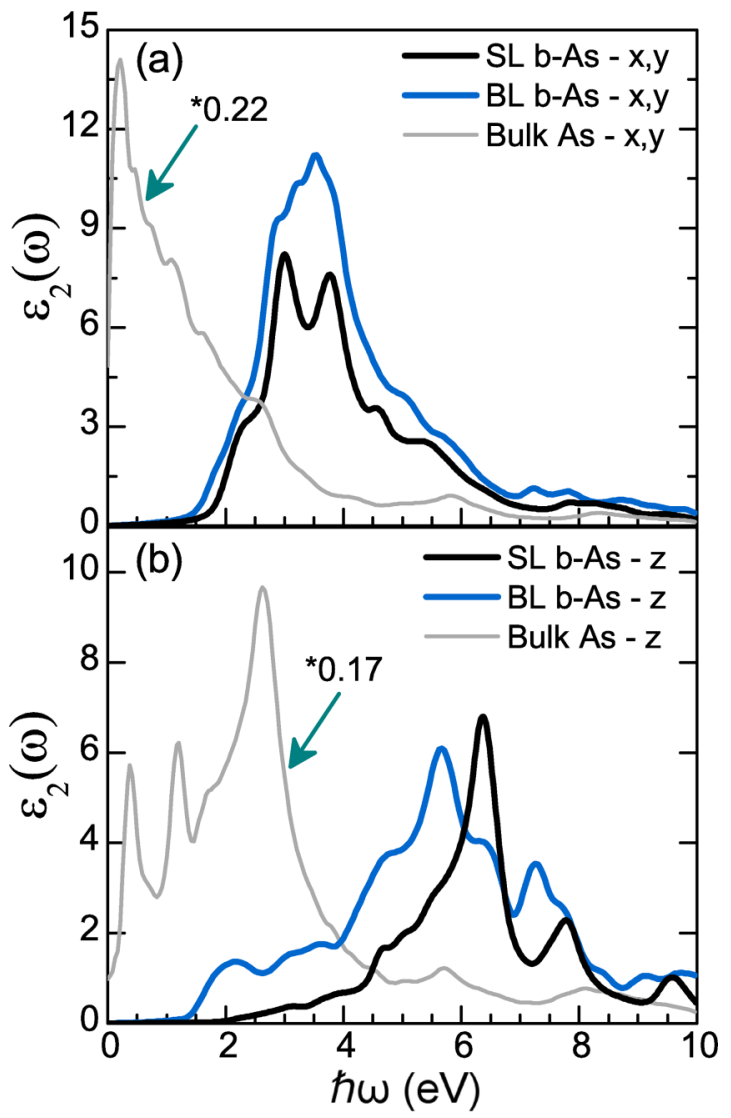

FIG. 5. The optical absorption spectra of bilayer buckled arsenene. Imaginary dielectric function $\varepsilon_{2}(\omega)$ of BL $b$-As with light polarized (a) along the $x$ and $y$ directions and (b) along the $z$ direction. PBE-RPA spectra of bilayer $b$-As are shown in blue lines, in comparison to that of SL $b$-As in black. Furthermore, $\varepsilon_{2}(\omega)$ of 3D gray As spectrum is shown in the background (intensity of spectrum is scaled accordingly for each case for the sake of comparison.)

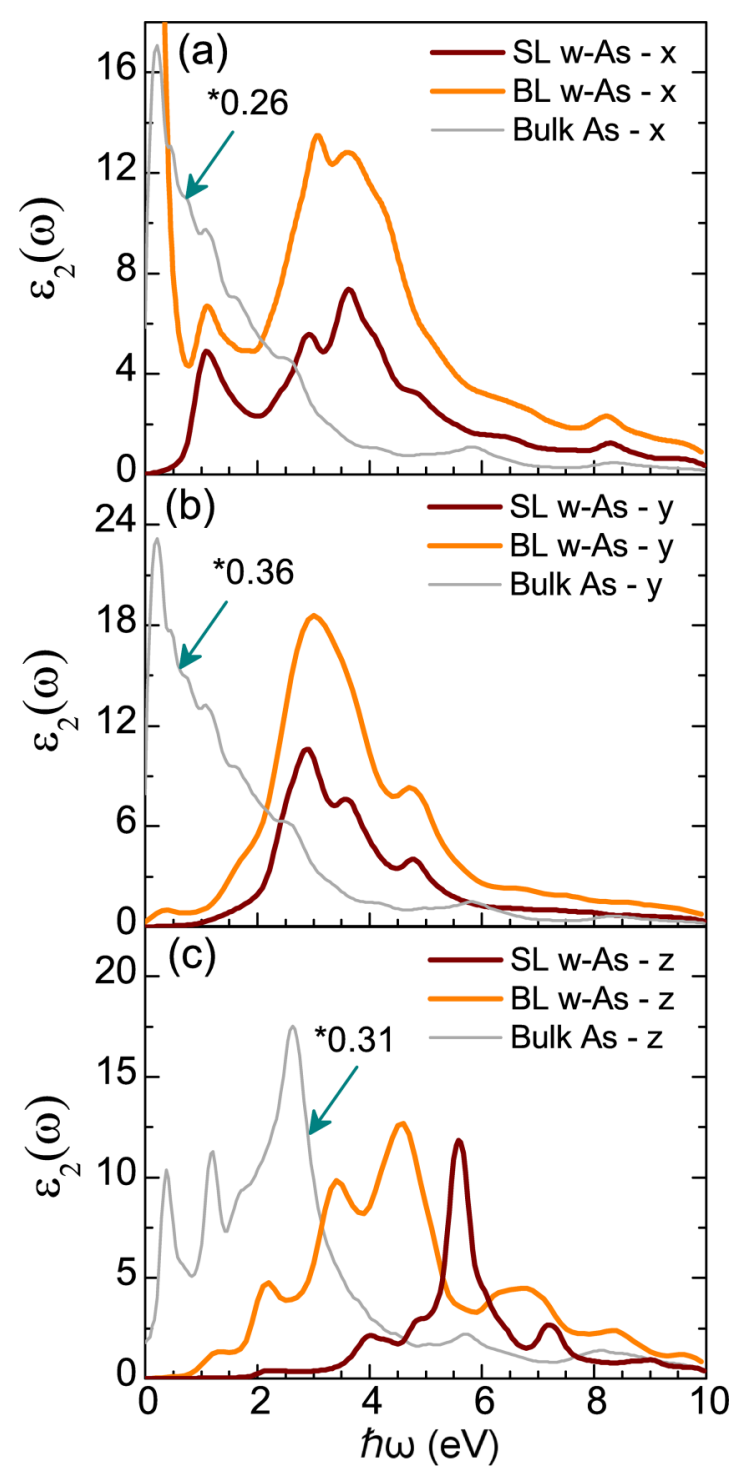

FIG. 6. The optical absorption spectra of bilayer washboard arsenene. Imaginary dielectric function $\varepsilon_{2}(\omega)$ of $\mathrm{BL} w$-As with light polarized (a) along the $x$ direction, (b) along the $y$ direction, and (c) along the $z$ direction. PBE-RPA spectra of bilayer $w$-As are shown in orange lines, in comparison to that of SL $w$-As in brown. Furthermore, $\varepsilon_{2}(\omega)$ of $3 \mathrm{D}$ gray As spectrum is shown in the background (intensity of spectrum is scaled accordingly for each case for the sake of comparison.)

spectra suggest enhanced properties within the visible and UV light range, in comparison to gray arsenic.

The layer-dependent absorbance of SL and BL As structures is also calculated and shown in Figs. 7 and 8 for the buckled and washboard phases, respectively. First, the layer-dependent absorbance is observed to increase upon increasing the number of layers, for $x$ and $y$ directions, $\sim$ twice up to $50 \%-60 \%$. Although gray arsenic's optical spectra display considerable differences from those of the few-layered phases, there is a relevant comparability in the sense of the line shape of the absorption coefficient spectrum. Additional optical absorption spectra (such as the optical conductivity, absorption coefficient, and electron energy loss) of SL, BL, and bulk 


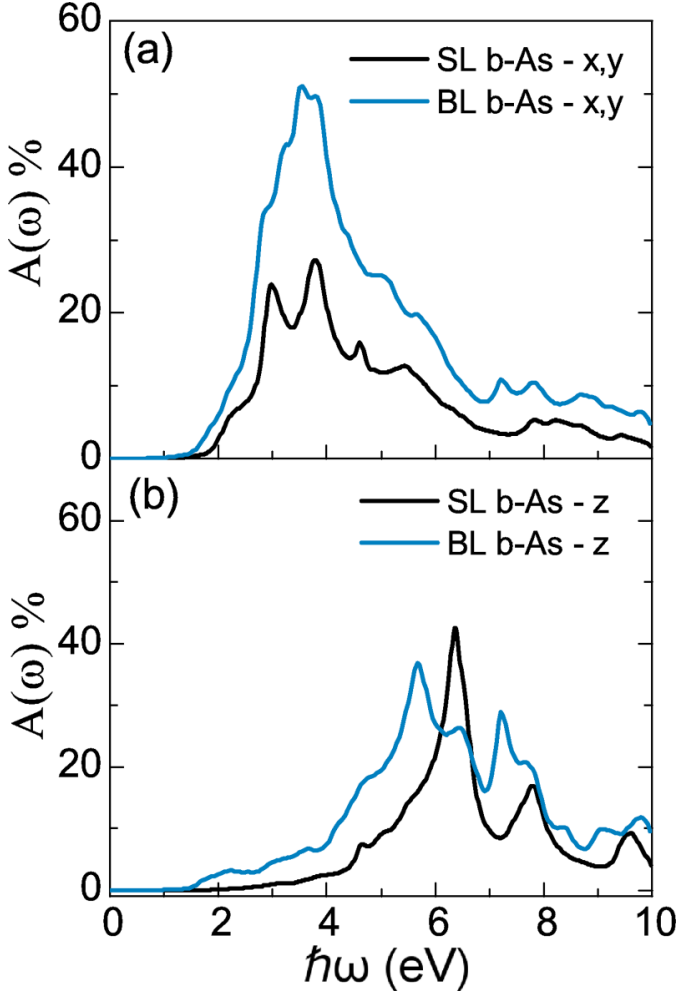

FIG. 7. Layer-dependent absorbance of SL and BL $b$-As: (a) light polarized along the $x$ and $y$ directions and (b) along the $z$ direction. SL $b$-As: black lines, BL $b$-As: blue lines.

As were calculated which we hope would be useful for applied research of the optoelectronic properties of group V 2D materials (see Supplemental Material [53]).

In summary, we report that the optical absorption is substantially red-shifted when the layer number is increased, for light polarized along the $z$ direction for both $b$-As and $w$-As, approaching the absorption spectrum of bulk As. Moreover, absorbance, which is a layer-dependent property, is enhanced for bilayer systems. A clear message that can be derived until now is that the wide energy window of absorption offered by tuning the number of layers and applying tensile strain can be utilized for various optoelectronic applications.

\section{EXCITONIC EFFECTS}

The electron-hole interactions within SL $b$ - and $w$-As are considered by employing the BSE, in order to calculate their many-body optical properties, as shown in Fig. 9. Accordingly, $b$-As reveals a sharp feature in its $G_{0} W_{0}$-BSE spectrum, especially around $3.2 \mathrm{eV}$, where the optical gap appears beyond $2.2 \mathrm{eV}$. It can be recalled that the prominent interband transitions, aside from the direct gap of $b$-As, occur also around the $\Gamma$ point beyond $\sim 4 \mathrm{eV}$, that are influential on the strong peak in the $G_{0} W_{0}$-BSE spectrum. However, the sharp absorption peak still falls beyond the quasiparticle indirect band gap of $2.64 \mathrm{eV}$. Moreover, the direct quasiparticle $G_{0} W_{0}$ gap of $b$-As was computed as $3.27 \mathrm{eV}$. According to the $G_{0} W_{0}$-BSE calculation for the buckled phase, the first peak appears at $2.57 \mathrm{eV}$, followed by a second smaller peak at $2.87 \mathrm{eV}$. Although both buckled and washboard phases have

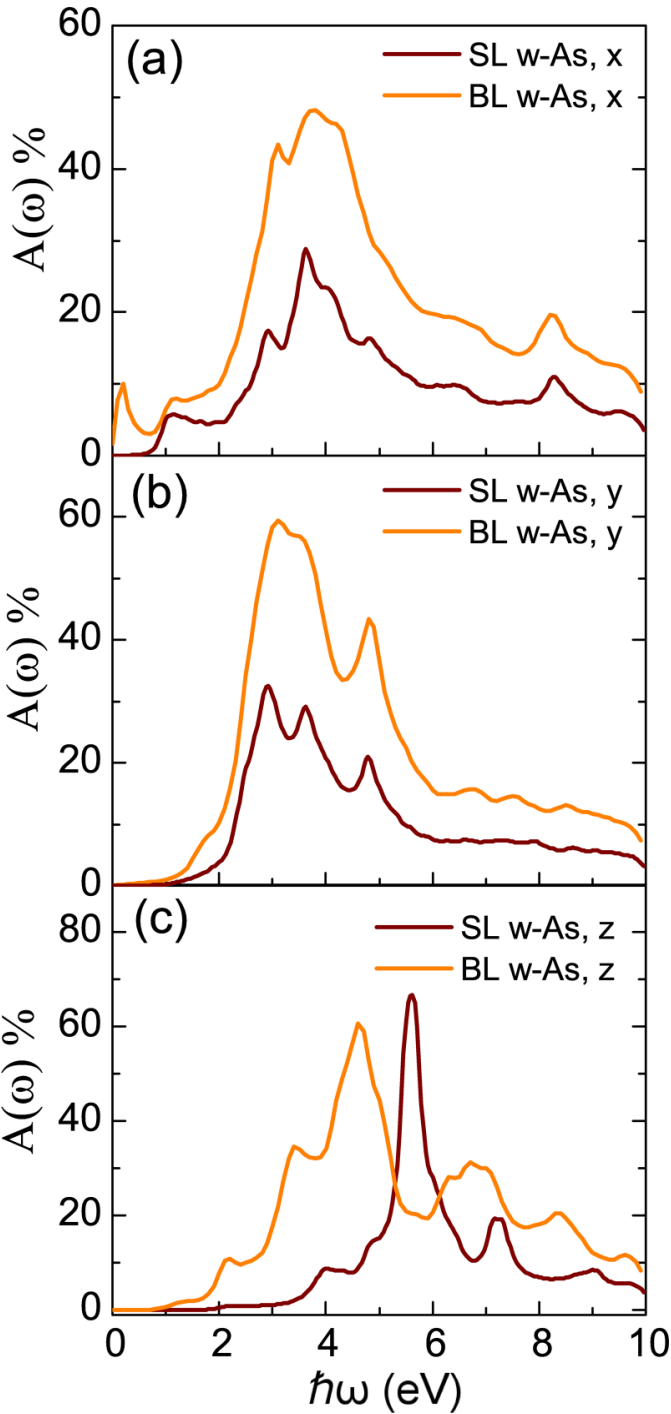

FIG. 8. Layer-dependent absorbance of SL and BL $w$-As: (a) light polarized along the $x$ direction, (b) along the $y$ direction, and (c) along the $z$ direction. SL $w$-As: brown lines, BL $w$-As: orange lines.

indirect band gaps, their corresponding direct gaps are shown by red vertical lines in Fig. 9. In order to identify the exciton binding energies, the vertical transitions are considered to account for the electron-hole interactions, which require taking the direct band gaps as reference. Hence, the exciton binding energy $\left(E_{b}\right)$ of the first peak in $b$-As is estimated to be around $700 \mathrm{meV}$. The smaller second peak suggests a binding energy of nearly $400 \mathrm{meV}$. Finally, the major peak in $G_{0} W_{0}$-BSE spectrum slightly falls behind the direct gap, pointing to a small binding of $\sim 60 \mathrm{meV}$. Yet, it should be minded that the $G_{0} W_{0}$-BSE spectrum could further be enhanced by increasing the number of $\mathbf{k}$ points and total bands in the calculation, which would presumably affect the resolution of the overall spectrum, i.e., the oscillator strengths, peak splittings, and positions.

For the $w$-As case, especially for polarization along the $y$ direction, one observes a distinguished peak near $3 \mathrm{eV}$. In reference to the quasiparticle direct band gap of $1.83 \mathrm{eV}$, there is no significant hint of a bound exciton for $w$-As along the $y$ direction since the main peak is blue-shifted quite beyond 

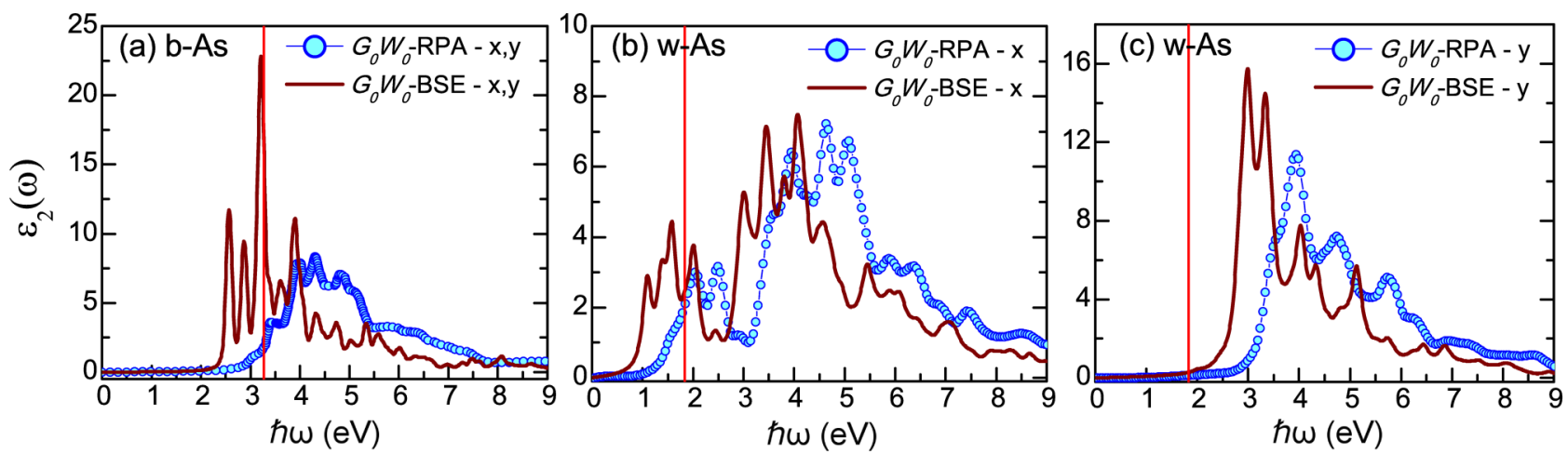

FIG. 9. The optical spectrum of SL As including the many-body effects are given in brown $\left(G_{0} W_{0}\right.$-BSE) and blue $\left(G_{0} W_{0}-\mathrm{RPA}\right)$ plots. Imaginary dielectric function $\varepsilon_{2}(\omega)$ vs $\hbar \omega$ of SL (a) $b$-As. (b) $w$-As for light polarized along the $x$ and (c) $y$ directions. The direct band gaps are shown by red vertical lines.

the quasiparticle gap of $w$-As. When incident light polarized along the $x$ direction, on the other hand, a small shoulder structure in $G_{0} W_{0}$-BSE absorption spectrum is observed near $1.1 \mathrm{eV}$, followed by a second shoulder near $1.4 \mathrm{eV}$ and fairly pronounced spectral weight near $1.58 \mathrm{eV}$, prior to the band gap. The difference between the direct band gap and the first peak is hence $\sim 730 \mathrm{meV}$. The peak feature slightly to the right-hand side of the shoulder points to a smaller binding of $\sim 250 \mathrm{meV}$.

The exciton binding energies of SL arsenene phases are hence comparable to those of few 2D semiconductors, which are on the order of a few hundred meV. Similar or slightly smaller exciton binding energies are given for $\mathrm{MoSe}_{2}, \mathrm{WS}_{2}$, and $\mathrm{WSe}_{2}$, which reveal $E_{b}$ as $450-500 \mathrm{meV}$ theoretically and 400-700 meV experimentally [56-59], as well as phosphorene $(0.70-0.80 \mathrm{eV})[11,60]$. Yet, $E_{b}$ of SL arsenene structures is fairly smaller than that of $2 \mathrm{D} \mathrm{MoS}_{2}(1.1 \mathrm{eV})$ [61]. To the best of our knowledge, no experimental or theoretical evidence of exciton binding in single-layer arsenene is available in the literature, apart from Ref. [62], which reports the exciton binding energy of bilayer arsenene as $\sim 500 \mathrm{meV}$. When combined with our results, this indicates that $E_{b}$ will decrease upon increasing the number of layers.

The aforementioned anisotropy of the optical properties of $w$-As is revealed once again regarding its excitonic properties for the different polarizations. Moreover, the line-shape difference between the $G_{0} W_{0}$-BSE and $G_{0} W_{0}$-RPA spectra is more drastic for the buckled structure than its washboard counterpart. In summary, for the buckled and one of the polarization directions of the washboard phase, SL arsenene possesses at least two bound excitons. For light polarized along the $y$ direction, $w$-As is found more eligible for continuous absorption across the solar spectrum. Hence, the anisotropy and variety in the structures of layered arsenene are also pronounced regarding their excitonic properties. The large modifications in the many-body optical properties of arsenene could allow for diverse photovoltaic and optoelectronic applications.

\section{CONCLUSIONS}

The arsenene phases have fundamental band gaps in the energy range which are suitable for $2 \mathrm{D}$ optoelectronic applications at room temperature. Additionally, width of the band gap, as well as the direct/indirect character, can be tuned by the number of layers and by applying biaxial and uniaxial expansive strain. Eventually, closing of the band gap and/or application of tensile strain can lead to insulator-metal transition for the layered arsenene phases [54]. In light of the electronic structure calculations, the strain- and layer-dependent optical properties of arsenene phases were computed by using stateof-the-art many-body first-principles methods. Due to the differing band gaps of SL $b$-As and $w$-As structures, their optical absorption onsets are also different. While the optical spectra usually point to an absorption within the visibleUV spectrum, the anisotropy of the washboard structure is noteworthy for functionalizing the optical properties of SL and BL arsenene. A systematical blue-shift of the optical absorption spectrum going from PBE to HSE to (QP) $G_{0} W_{0}$ calculations within RPA has been observed. Furthermore, the layer-dependent absorbance increased as the layer number was raised. Also, the biaxial and uniaxial in-plane tensile strains usually red-shifted the optical spectra, except for strain applied along rectangular lattice vector a of $w$-As.

Finally, the optical response of SL As was substantially affected by accounting for the electron-hole interactions. Approximate first-exciton binding energies of $b$-As and $w$-As for the $y$ direction are estimated to be on the order of $700 \mathrm{meV}$. The directional dependence of the many-body optical properties is also observed for the BSE calculations of SL arsenene. In summary, the band gap, optical absorption onset, and exciton binding energies can be tuned by either adding more layers, polarizing light along differing directions in the lattice, or applying strain.

\section{ACKNOWLEDGMENTS}

The computing resources are provided by SVR High Performance Computing Center, TUBITAK ULAKBIMHigh Performance and Grid Computing Center (TR-Grid eInfrastructure) and the National Center for High Performance Computing of Turkey (UHeM) under Grant No. 5003622015. This work was supported by the Scientific and Technological Research Council of Turkey (TUBITAK) under Project No. 115F088. E.D. acknowledges the support from The Turkish Academy of Sciences within Outstanding Young Scientists Award Program (TUBA-GEBIP). S.C. acknowledges financial support from Turkish Academy of Sciences (TUBA). 
[1] A. Splendiani, S. Liang, Z. Yuanbo, L. Tianshu, K. Jonghwan, C.-Y. Chim, G. Galli, and F. Wang, Nano Lett. 10, 1271 (2010).

[2] K. F. Mak, C. Lee, J. Hone, J. Shan, and T. F. Heinz, Phys. Rev. Lett. 105, 136805 (2010).

[3] Q. H. Wang, K. Kalantar-Zadeh, A. Kis, J. N. Coleman, and M. S. Strano, Nat. Nanotechnol. 7, 699 (2012).

[4] S. Cahangirov, M. Topsakal, E. Aktürk, H. Sahin, and S. Ciraci, Phys. Rev. Lett. 102, 236804 (2009).

[5] B. Feng, Z. Ding, S. Meng, Y. Yao, X. He, P. Cheng, L. Chen, and K. Wu, Nano Lett. 12, 3507 (2012).

[6] C. Jin, F. Lin, K. Suenaga, and S. Iijima, Phys. Rev. Lett. 102, 195505 (2009).

[7] Y. Li, Y. Rao, K. F. Mak, Y. You, S. Wang, C. R. Dean, and T. F. Heinz, Nano Lett. 13, 3329 (2013).

[8] Z. Y. Al Balushi, K. Wang, R. K. Ghosh, R. A. Vila, S. M. Eichfeld, J. D. Caldwell, X. Qin, Y. C. Lin, P. A. DeSario, G. Stone, S. Subramanian et al., Nat. Mater. (to be published).

[9] L. Li, Y. Yu, G. J. Ye, Q. Ge, X. Ou, H. Wu, D. Feng, X. H. Chen, and Y. Zhang, Nat. Nanotechnol. 9, 372 (2014).

[10] H. Liu, A. T. Neal, Z. Zhu, Z. Luo, X. Xu, D. Tomanek, and P. D. Ye, ACS Nano 8, 4033 (2014).

[11] V. Tran, R. Soklaski, Y. Liang, and L. Yang, Phys. Rev. B 89, 235319 (2014).

[12] A. Chaves, T. Low, P. Avouris, D. Cakir, and F. M. Peeters, Phys. Rev. B 91, 155311 (2015).

[13] V. O. Özçelik, O. Ü. Aktürk, E. Durgun, and S. Ciraci, Phys. Rev. B 92, 125420 (2015).

[14] Z. Zhu and D. Tomanek, Phys. Rev. Lett. 112, 176802 (2014).

[15] C. Kamal and M. Ezawa, Phys. Rev. B 91, 085423 (2015).

[16] O. Ü. Aktürk, V. O. Özçelik, and S. Ciraci, Phys. Rev. B 91, 235446 (2015).

[17] Z. Zhang, Z. Yan, Y. Li, Z. Chen, and H. Zeng, Angew. Chem. Int. Ed. 54, 3112 (2015).

[18] E. Aktürk, O. Ü. Aktürk, and S. Ciraci, Phys. Rev. B 94, 014115 (2016).

[19] Z. Zhang, J. Xie, D. Yang, Y. Wang, M. Si, and D. Xue, Appl. Phys. Express 8, 055201 (2015).

[20] S. Y. Ma, P. Zhou, L. Z. Sun, and K. W. Zhang, Phys. Chem. Chem. Phys. 18, 8723 (2016).

[21] Z. Zhu, J. Guan, and D. Tomanek, Phys. Rev. B 91, 161404 (2015)

[22] L. Kou, Y. Ma, X. Tan, T. Frauenheim, A. Du, and S. Smith, J. Phys. Chem. C 119, 6918 (2015).

[23] K. Luo, S. Y. Chen, and C. G. Duan, Sci China-Phys. Mech. Astron. 58, 087301 (2015).

[24] Y. Wang and Y. Ding, J. Phys.: Condens. Matter 27, 225304 (2015).

[25] H. Zhang, Y. Ma, and Z. Chen, Nanoscale 7, 19152 (2015).

[26] C. Wang, Q. Xia, Y. Nie, M. Rahman, and G. Guo, AIP Advances 6, 035204 (2016).

[27] F. Ersan, E. Aktürk, and S. Ciraci, J. Phys. Chem. C 120, 14345 (2016).

[28] W. Kohn, and L. J. Sham, Phys. Rev. 140, A1133 (1965).

[29] H. S. Tsai, S. W. Wang, C. H. Hsiao, C. W. Chen, H. Ouyang, Y. L. Chueh, H. C. Kuo, and J. H. Liang, Chem. Mater. 28, 425 (2016).

[30] P. E. Blöchl, Phys. Rev. B 50, 17953 (1994).
[31] M. Gajdoš, K. Hummer, G. Kresse, J. Furthmüller, and F. Bechstedt, Phys. Rev. B 73, 045112 (2006).

[32] G. Kresse and J. Hafner, Phys. Rev. B 47, 558 (1993).

[33] G. Kresse and J. Hafner, Phys. Rev. B 49, 14251 (1994).

[34] G. Kresse and J. Furthmüller, Comput. Mater. Sci. 6, 15 (1996).

[35] G. Kresse and J. Furthmüller, Phys. Rev. B 54, 11169 (1996).

[36] J. P. Perdew, K. Burke, and M. Ernzerhof, Phys. Rev. Lett. 77, 3865 (1996).

[37] J. P. Perdew, K. Burke, and M. Ernzerhof, Phys. Rev. Lett. 78, 1396 (1997).

[38] S. J. Grimme, Comput. Chem. 27, 1787 (2006).

[39] T. Bucko, J. Hafner, S. Lebegue, and J. G. Angyan, J. Phys. Chem. A 114, 11814 (2010).

[40] J. Paier, M. Marsman, K. Hummer, G. Kresse, I. C. Gerber, and J. G. Angyan, J. Chem. Phys. 124, 154709 (2006).

[41] J. Heyd, G. E. Scuseria, and M. Ernzerhof, J. Chem. Phys. 118, 8207 (2003); 124, 219906 (2006).

[42] J. Heyd and G. E. Scuseria, J. Chem. Phys. 120, 7274 (2004); 121, 1187 (2004).

[43] L. Hedin, Phys. Rev. 139, A796 (1965).

[44] M. S. Hybertsen and S. G. Louie, Phys. Rev. B 34, 5390 (1986).

[45] M. Shishkin and G. Kresse, Phys. Rev. B 74, 035101 (2006).

[46] D. Bohm and D. Pines, Phys. Rev. 82, 625 (1951); 85, 338 (1952); 92, 609 (1953).

[47] H. Ehrenreich and M. H. Cohen, Phys. Rev. 115, 786 (1959).

[48] H. J. Monkhorst and J. D. Pack, Phys. Rev. B 13, 5188 (1976).

[49] E. E. Salpeter and H. A. Bethe, Phys. Rev. 84, 1232 (1951).

[50] S. Albrecht, L. Reining, R. Del Sole, and G. Onida, Phys. Rev. Lett. 80, 4510 (1998).

[51] M. Rohlfing and S. G. Louie, Phys. Rev. Lett. 81, 2312 (1998).

[52] L. X. Benedict, E. L. Shirley, and R. B. Bohn, Phys. Rev. Lett. 80, 4514 (1998).

[53] See Supplemental Material at http://link.aps.org/supplemental/ 10.1103/PhysRevB.94.205410 for a description of the further spectral optical functions such as the layer-dependent absorbance $[A(\omega)]$, optical conductivity $[\sigma(\omega)]$, absorption coefficient $[\alpha(\omega)]$ and electron energy-loss function $\left[-\operatorname{Im}\left(\varepsilon^{-1}\right)\right]$. The calculated spectra are displayed for the single-layer, bilayer and bulk forms of arsenene, considering both buckled and washboard phases. Finally, a comparison of the SOC effect excluded and included bandstructures and optical spectra are given.

[54] D. Kecik, E. Durgun, and S. Ciraci, Preceeding paper Phys. Rev. B 94, 205409 (2016).

[55] M. Buscema, D. J. Groenendijk, S. I. Blanter, G. A. Steele, H. S. J. van der Zant, and A. Castellanos-Gomez, Nano Lett. 14, 3347 (2014).

[56] T. C. Berkelbach, M. S. Hybertsen, and D. R. Reichman, Phys. Rev. B 88, 045318 (2013).

[57] M. M. Ugeda, A. J. Bradley, S.-F. Shi, F. H. da Jornada, Y.Zhang, D. Y. Qiu, W. Ruan, S.-K. Mo, Z. Hussain, Z.-X. Shen, F. 
Wang, S. G. Louie, and M. F. Crommie, Nat. Mater. 13, 1091 (2014).

[58] Z. Ye, T. Cao, K. O’Brien, H. Zhu, X. Yin, Y. Wang, S. G. Louie, and X. Zhang, Nature (London) 513, 214 (2014).

[59] K. He, N. Kumar, L. Zhao, Z. Wang, K. F. Mak, H. Zhao, and J. Shan, Phys. Rev. Lett. 113, 026803 (2014).
[60] D. Cakir, C. Sevik, and F. M. Peeters, Phys. Rev. B 92, 165406 (2015).

[61] H.-P. Komsa and A. V. Krasheninnikov, Phys. Rev. B 86, 241201(R) (2012).

[62] A. Chaves, M. Z. Mayers, F. M. Peeters, and D. R. Reichman, Phys. Rev. B 93, 115314 (2016). 